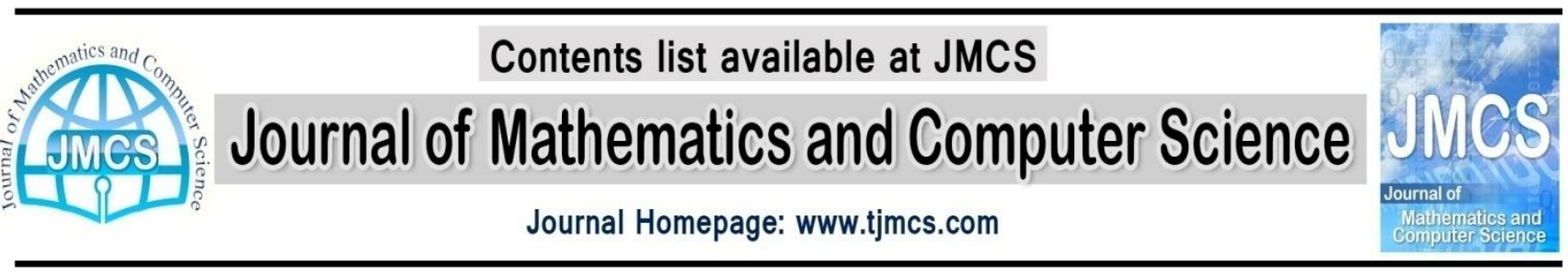

\title{
Using shifted Legendre polynomials for solving optimal control problem of an HIV infection treatment control model
}

Article history:

Received July 2013

Accepted August 2013

Available online August 2013

\author{
M. Alizadehjamal ${ }^{1}$, M. H. Farahi' ${ }^{2}$ 'S. A. Mahdipour ${ }^{3}$ \\ ${ }^{1}$ Islamic Azad University, Mashhad Baranch, Mashhad, Iran \\ ${ }^{2}$ Ferdowsi University, Mashhad, Iran \\ ${ }^{3}$ Hakim Sabzevari University, Sabzevar, Iran \\ E-mail: M_alizadehjamal@yahoo.com
}

\begin{abstract}
In this paper we introduce a numerical technique based on Legendre polynomials for solving of nonlinear optimal control problems, where this approach is used for solving optimal control problem of an HIV infection treatment control model. In this paper, first by using healthy cells $\mathrm{CD} 4^{+} \mathrm{T}(\mathrm{T})$, infected cells $\mathrm{CD}^{+}{ }^{+} \mathrm{T}(\mathrm{I})$, viral load (V) and also by using a drug inhibitor of reverse polygraph as a control function, a control model is presented for treatment of HIV infection. A cost function to minimize the cost of drug during the treatment is defined as well. To find the pair of trajectory and control of such nonlinear optimal control problem, we used shifted Legendre polynomials to approximate optimal pair of trajectory and control.
\end{abstract}

Keywords: Legendre polynomials, HIV infection, Optimal control.

\section{Introduction}

Despite remarkable success in medical science, still solution and definitive treatment for HIV has not been found by scientists, and every day is added to statistics victims and patients. The importance of these diseases caused, the other researchers including mathematic scientists, to deal with it, come to the help of medical science scientists. In this regard, various mathematical models to represent the dynamics of immune system cells and the HIV virus, has been presented. Solving optimal control problems related to these models, because of the nonlinear dynamic system, the classic method of solving the optimal control is not possible. Therefore present a simple and efficient method for solving such problems is very important. In recent years approximation functions, such as orthogonal functions and polynomials to solve a variety of mathematical and dynamical systems are used. Orthogonal functions and polynomial series have received considerable attention in dealing with various problems 
of dynamical systems. Examples are the use of the Fourier series [1, 2], the Walsh functions [3], the Taylor series [4], the Chebyshev polynomials [5] and the block- pulse functions [6].

In this paper we described the optimal control model of HIV will be solved by shifted-Legendre polynomials

\section{Theory}

\subsection{Function approximation}

A function $f(t) \in L^{2}\left[0, t_{f}\right]$ can be approximated as:

$$
f(t)=\sum_{i=0}^{\infty} f_{i} P_{i}(t)
$$

In practice we only consider a finite number of terms, that is

$$
\sum_{i=0}^{m-1} f_{i} P_{i}(t) \approx f(t)
$$

The coefficient of shifted-Legendre polynomial $f_{i}$ can be obtained using:

$$
f_{i}=\frac{2 i+1}{t_{f}} \int_{0}^{t_{f}} f(\tau) P_{i}(\tau) d \tau
$$

Equation (2) can be written in a matrices form as:

$$
F^{T} P(t) \approx f(t) \quad \& \quad P(t)^{T} F \approx f(t)
$$

Where $\mathrm{F}$ and $\mathrm{P}(\mathrm{t})$ are $\mathrm{m} \times 1$ matrices which are given by:

$$
\begin{aligned}
& F=\left[\begin{array}{lllll}
f_{0} & f_{1} & f_{2} & \ldots & f_{m-1}
\end{array}\right]^{T} \\
& P(t)=\left[\begin{array}{llllll}
P_{0} & P_{1} & P_{2} & \ldots & P_{m-1}
\end{array}\right]^{T} .
\end{aligned}
$$

\section{2. The Operational Matrix of Integration}

Integration of the vector $\mathrm{P}(\mathrm{t})$ defined in Eq. (4) can be written as:

$$
\int_{0}^{t} P(s) d s=H_{m \times m} P(t)_{m \times 1}
$$

Using Esq. (3, 5):

$$
\begin{aligned}
& \int_{0}^{t} f(s) d s=\int_{0}^{t} F^{T} P(s) d s=F^{T} H P(t) \\
& \int_{0}^{t} f(s) d s=\int_{0}^{t} P(s)^{T} F d s=P(t)^{T} H^{T} F
\end{aligned}
$$

Where the matrix $\mathrm{H}$ is obtained as follows [4]: 


$$
H=t_{f}\left[\begin{array}{cccccc}
\frac{1}{2} & \frac{1}{2} & 0 & 0 & \ldots & 0 \\
-\frac{1}{6} & 0 & \frac{1}{6} & 0 & \ldots & 0 \\
0 & -\frac{1}{10} & 0 & \frac{1}{10} & \ldots & 0 \\
0 & 0 & -\frac{1}{14} & 0 & \ldots & \vdots \\
\vdots & \vdots & \vdots & \vdots & \ldots & \frac{1}{2}(2 m-3) \\
0 & 0 & 0 & \cdots & -\frac{1}{2}(2 m-3) & 0
\end{array}\right]_{m \times m}
$$

\section{3. The product Operational Matrix}

The product operational matrix $F$ can be defined as follows [7]:

$$
P(t) P^{T}(t) F \approx F P(t)
$$

For example, for $\mathrm{m}=3$ the square matrix $F$ can be defined as follows:

$$
F=\left[\begin{array}{ccc}
f_{0} & f_{1} & f_{2} \\
\frac{1}{3} f_{1} & f_{0}+\frac{2}{5} f_{2} & \frac{2}{3} f_{1} \\
\frac{1}{5} f_{2} & \frac{2}{5} f_{1} & f_{0}+\frac{2}{7} f_{2}
\end{array}\right]_{3 \times 3}
$$

Where $\mathbf{F}^{\mathrm{T}}=\left[\begin{array}{lll}\mathbf{f}_{\mathbf{0}} & \mathbf{f}_{\mathbf{1}} & \mathbf{f}_{\mathbf{2}}\end{array}\right]$, is vector of coefficient of shifted Legendre polynomials.

\section{4. HIV Basic Model}

The target cells of HIV infection are lymphocyte helper cells, especially $C D 4^{+} T$ cells. These cells become infected and begin to produce free various. The main fact about HIV infection is reducing the count of $\mathrm{CD} 4^{+} \mathrm{T}$ cells, which have an essential role in protecting body against deferent pathogens. So it is important to understand the dynamics of $\mathrm{CD}^{+}{ }^{+} \mathrm{T}$ cell count as a function of time. In HIV infection basic model, three groups of molecules are considered; Uninfected $C D 4^{+} T$ cells $(T)$, infected $C D 4^{+} T$ cells (I) and viral load (V). Biological descriptions, translation to reactions and corresponding ODE's are presented in Table 1. 
Table 1. HIV basic model interactions.

\begin{tabular}{lllc}
\hline Biological description & $\begin{array}{l}\text { Translation to } \\
\text { reactions }\end{array}$ & Reaction rate & Translation to ODE \\
\hline CD4 ${ }^{+} \mathrm{T}$ cells production & $0 \rightarrow \mathrm{T}$ & $\mathrm{s}$ & $\dot{\mathrm{T}}=\mathrm{s}$ \\
$\mathrm{CD}^{+} \mathrm{T}$ cells natural death & $\mathrm{T} \rightarrow 0$ & $\mathrm{~d}$ & $\mathrm{~T}=-\mathrm{dT}$ \\
$\mathrm{CD}^{+} \mathrm{T}$ cells become infected by virus $\mathrm{T}+\mathrm{V} \rightarrow \mathrm{I}+\mathrm{V}$ & $\beta$ & $\dot{\mathrm{T}}=-\beta \mathrm{TV} \quad \mathrm{I}=\beta T V$ \\
Infected CD4 ${ }^{+} \mathrm{T}$ cells death & $\mathrm{I} \rightarrow 0$ & $\mu$ & $\dot{\mathrm{I}}=-\mu \mathrm{l}$ \\
Virus replication in infected $\mathrm{CD} 4^{+} \mathrm{T}$ & $\mathrm{I} \rightarrow \mathrm{I}+\mathrm{V}$ & $\mathrm{k}$ & $\dot{\mathrm{V}}=\mathrm{kl}$ \\
Virus natural death & $\mathrm{V} \rightarrow 0$ & $\mathrm{c}$ & $\dot{\mathrm{V}}=-\mathrm{cV}$
\end{tabular}

Now, according to Table 1, the complete ODE model, which is sum of contributions from all reactions, is as follows:

$$
\begin{aligned}
T & =s-d T-\beta T V \\
\square & =\beta T V-\mu I \\
V & =k I-c V
\end{aligned}
$$

Where the following estimated parameters are as model (1) [8]:

$s=7, d=0.007, \beta=0.00000042163, \mu=0.0999, c=0.2, k=90.67$

\section{5. HIV infection treatment control model}

There are three convenient groups of drugs for AIDS retroviral therapy; reverse transcriptase, protease, and Integrate enzyme inhibitors. In this section, we study the role of reverse transcriptase inhibitors. The main action of this kind of drugs is preventing to produce viral load with infection Lymphocyte cells. This action is equivalent to the reaction $\boldsymbol{I} \rightarrow \boldsymbol{I}+\boldsymbol{V}$ [8]. So we control the third equation to prevent of produce viral load with infection lymphocyte cells. This control function is called $u(t)$, where $\mathbf{0} \leq \boldsymbol{u}(\boldsymbol{t}) \leq$ 1. The most drug efficiency is in the case $\boldsymbol{u}(\boldsymbol{t}) \equiv \mathbf{1}$ which means viral load is not produce by infection cells. At the other side, $\boldsymbol{u}(\boldsymbol{t}) \equiv \mathbf{0}$ is the case which the drug does not change the disease progression. By above argument, the control system is as:

$$
\begin{aligned}
T & =s-d T-\beta T V \\
I & =\beta T V-\mu I \\
V & =k I(1-u)-c V
\end{aligned}
$$

\section{Solving HIV infection treatment control model}

With Using [9], consider the objective functional to be defined as:

$$
J(T, I, V, u)=\int_{t_{0}}^{t_{f}}\left[T(t)-\frac{1}{2} \alpha u^{2}(t)\right] d t
$$

Where $\boldsymbol{\alpha}=\mathbf{1 1 0}$. Our goal is maximizing the objective functional J subject to the control system (9); that is, maximizing the total count of $\mathrm{CD}^{+}{ }^{+} \mathrm{T}$ cells and minimizing the costs of treatment by applying some RTI 
drugs. The solution of this optimal control problem should be calculated by numerical methods. We have used a special discretization method, based on shifted Legendre polynomials. For detailed explanation of this method, see [7]. So optimal control problem in $[\mathbf{0 , 1 0 0 0 ]}$ interval days, given by:

$$
\begin{array}{ll}
\max & J=\int_{0}^{1000}\left[T(t)-\frac{1}{2} \alpha u^{2}(t)\right] d t \\
s . t . & T=s-d T-\beta T V \\
& I=\beta T V-\mu I \\
& V=k I(1-u)-c V \\
& T(t), I(t), V(t) \geq 0,0 \leq u(t) \leq 1, \forall t \in[0,1000] \\
& T(0)=363, I(0)=57, V(0)=28860
\end{array}
$$

In this problem using of drug after 129 days of entry the HIV virus into the body has been considered. Therefore, the initial value of problem to the solution of AVK discrete optimization technique for modelbased HIV infection has been calculated at 129 days [8]. Constraints optimal control problem are as a nonlinear differential equations. These constraints can be convert to nonlinear algebraic equations which includes a system of 9 equations and 12 unknown with using shifted Legendre polynomials. Using this approximation method, the first nonlinear constraint of problem, can be transformed to non-linear algebraic system of three equations as follows:

$4.5 T_{0}-7 T_{2}+0.0002 T_{0} V_{0}-0.0002 T_{1} V_{1}-0.0008 T_{2} V_{2}-0.0004 T_{0} V_{2}-0.0004 T_{2} V_{0}+0.0008 T_{1} V_{2}+0.0008 T_{2} V_{1}-4500=0$

$3.5 T_{0}+T_{1}-7 T_{2}+0.0002 T_{0} V_{0}-0.0002 T_{1} V_{1}-0.0008 T_{2} V_{2}-0.0004 T_{0} V_{2}-0.0004 T_{2} V_{0}+0.0008 T_{1} V_{2}+0.0008 T_{2} V_{1}-3500=0$

$T_{1}-2.5 T_{2}+0.00007 T_{0} V_{1}+0.00007 T_{1} V_{0}-0.0002 T_{0} V_{2}-0.0002 T_{2} V_{0}+0.0003 T_{1} V_{2}+0.0003 T_{2} V_{1}-0.0001 T_{1} V_{1}-0.0004 T_{2} V_{2}=0$

So the second constraint of problem makes nonlinear algebraic equations for the following:

$51 I_{0}-100 I_{2}-0.0002 T_{0} V_{0}+0.0002 T_{1} V_{1}+0.0008 T_{2} V_{2}+0.0004 T_{0} V_{2}+0.0004 T_{2} V_{0}-0.0008 T_{1} V_{2}-0.0008 T_{2} V_{1}=0$

$I_{1}+50 I_{0}-100 I_{2}-0.0002 T_{0} V_{0}+0.0002 T_{1} V_{1}+0.0008 T_{2} V_{2}+0.0004 T_{0} V_{2}+0.0004 T_{2} V_{0}-0.0008 T_{1} V_{2}-0.0008 T_{2} V_{1}=0$

$17 I_{1}-49 I_{2}-0.00007 T_{0} V_{1}-0.00007 T_{1} V_{0}+0.0002 T_{0} V_{2}+0.0002 T_{2} V_{0}-0.0003 T_{1} V_{2}-0.0003 T_{2} V_{1}-0.0001 T_{1} V_{1}-0.0004 T_{2} V_{2}=0$

The following non-linear algebraic equations are obtained by the third constraint of problem:

$101 V_{0}-200 V_{2}-45335 I_{0}+90670 I_{2}+45335 I_{0} u_{0}-45335 I_{1} u_{1}-181340 I_{2} u_{2}-90670 I_{0} u_{2}-90670 I_{2} u_{0}+181340 I_{1} u_{2}+181340 I_{2} u_{1}-28860=0$

$100 V_{0}+V_{1}-200 V_{2}-45335 I_{0}+90670 I_{2}+45335 I_{0} u_{0}-45335 I_{1} u_{1}-181340 I_{2} u_{2}-90670 I_{0} u_{2}-90670 I_{2} u_{0}+181340 I_{1} u_{2}+181340 I_{2} u_{1}=0$

$33 V_{1}-99 V_{2}-15142 I_{1}+45335 I_{2}+15142 I_{0} u_{1}+15142 I_{1} u_{0}-45335 I_{0} u_{2}-45335 I_{2} u_{0}+60477 I_{1} u_{2}+60477 I_{2} u_{1}-30193 I_{1} u_{1}-75528 I_{2} u_{2}=0$

Where

$$
\begin{aligned}
& T(t) \square\left[\begin{array}{lll}
T_{0} & T_{1} & T_{2}
\end{array}\right] P(t)_{3 \times 1} \\
& I(t) \square\left[\begin{array}{lll}
I_{0} & I_{1} & I_{2}
\end{array}\right] P(t)_{3 \times 1} \\
& V(t) \square\left[\begin{array}{lll}
V_{0} & V_{1} & V_{2}
\end{array}\right] P(t)_{3 \times 1} \\
& P(t)=\left[\begin{array}{lll}
P_{0}(t) & P_{1}(t) & P_{2}(t)
\end{array}\right]^{T}
\end{aligned}
$$

The objective function of the problem (10) can be written as follows by nonlinear algebraic function coefficients in $[0,1000]$ days with terms of shifted Legendre polynomials:

$$
J \approx 1000 T_{0}-55000 u_{0}^{2}-55000 u_{0} u_{1}-18315 u_{1}^{2}-18370 u_{1} u_{2}-18315 u_{2}^{2}
$$


Now the objective function of equation (14), equations (11), (12) and (13) that are related to constraints of problem, the optimal control problem (10) can be wrote by nonlinear program problem. With solving of this nonlinear programming problem by Mathematica software, $\mathrm{T}(\mathrm{t}), \mathrm{l}(\mathrm{t}), \mathrm{V}(\mathrm{t})$ and $\mathrm{u}(\mathrm{t})$ functions are obtained as following form:

$$
\begin{aligned}
& T(t) \square-0.0013 t^{2}+2 t+363, \\
& I(t) \square-0.0001 t^{2}+0.0002 t+57, \\
& V(t) \square-0.6 t^{2}+6 t+28860, \\
& u(t) \square 0.6
\end{aligned}
$$

By plotting these functions, the following diagrams are obtained:
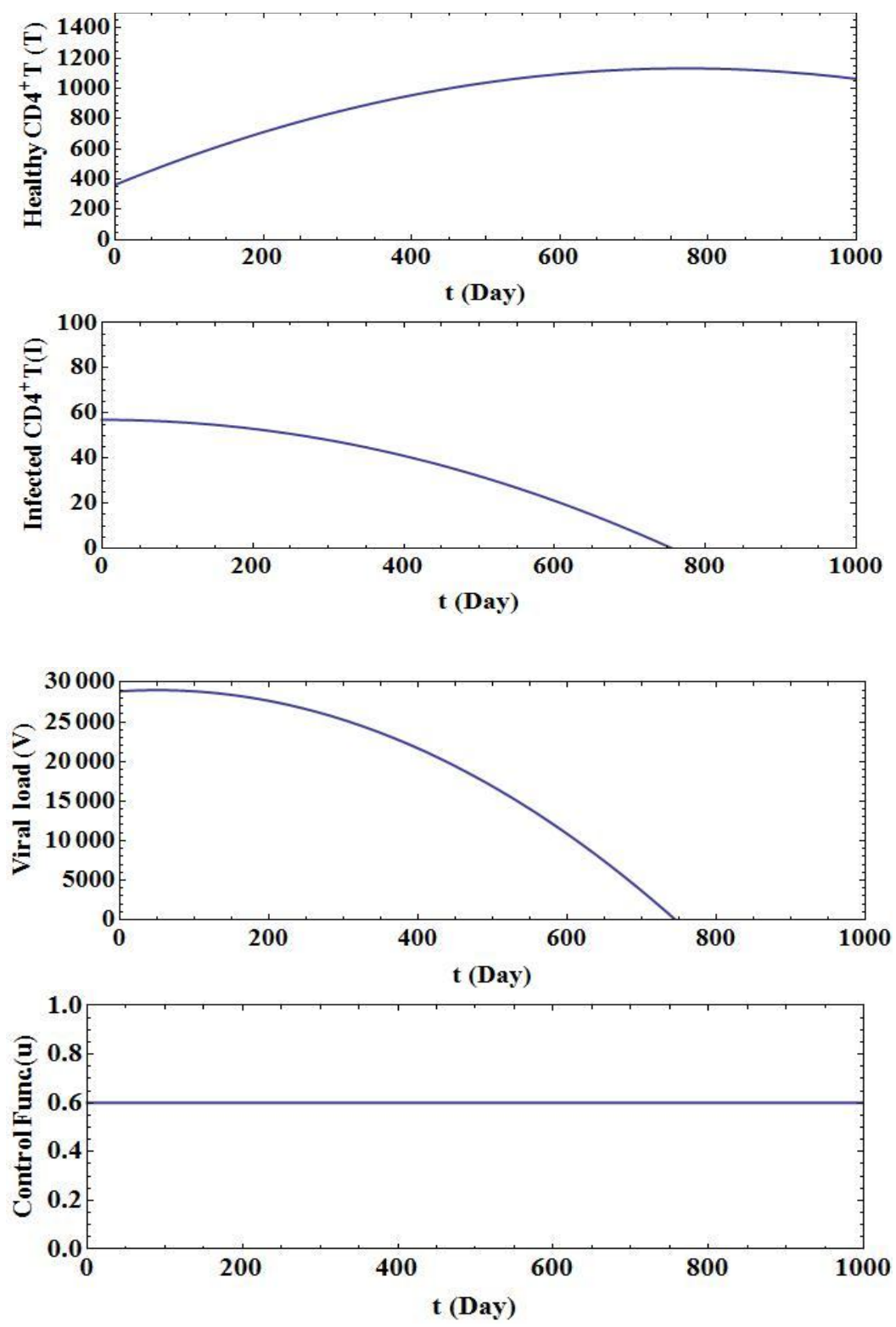
Figure. 1 Solution of the optimal control problem with control

According to Figure 1, it is seen that after taking the drug, the number of uninfected cells $C D 4^{+} T(\mathrm{~T})$ is increasing, however these cells rate of increasing after 200 days because of approaching normal human body decreases. The number of infected cells (I) and viral load (V) is reduced and finally, an average dose for the drug during treatment recommended.

\section{Conclusions}

According to, the dynamic of most real systems in nature are nonlinear and optimal control problems related to them in classical form are often extremely complex and difficult, method used in this paper is effective and efficient method, because it makes these problems to nonlinear algebraic problems solving. In this paper, the problem of minimizing the cost of treatment with drug in a model controlling HIV infection is solved with using Shifted Legendre polynomials and a mean value for drug use during treatment is recommended. Figures obtained from the solution of the problem show drug effects during treatment as well as.

\section{References}

[1] B. A. Ardekani, and A. Keyhani, "Identification of non-linear systems using the exponential Fourier series," Int. J. Control, vol. 50, pp. 1553-1558, 1989.

[2] S. Dong-Her, and K. Fan-Chu, "Analysis and parameter estimation of a scaled system via shifted Legender polynomials," International Journal of systems Science, vol. 17, pp. 401-408, 1986.

[3] W. L. Chen, and Y. P. Shih, "Parameter estimation of bilinear systems via Walsh functions," Journal of the Franklin Institute, vol. 305, pp. 249-257, 1978.

[4] M. Gulsu, and M. Sezer, "A Taylor polynomial approach for solving differential-difference equations," Journal of Computational and Applied Mathematics, vol. 186, pp. 349-364, 2006.

[5] H. Jaddu, and E. Shimomura, "Solution of nonlinear optimal control problem using Chebyshev polynomials," Proceeding of $2^{\text {nd }}$ Asian Control Conference, pp. 417-420, 1997.

[6] K. Maleknejad, M. Shahrezaee, and H. Khatami, "Numerical solution of integral equations system of the second kind by block-pulse functions," Applied Mathematics and Computation, vol. 166, pp. 1524, 2005.

[7] M. Razzaghi, and S. Yousefi, "Legender wavelets method for the solution of nonlinear problems in the calculus of variations," Mathematical and Computer Modelling, vol. 34, pp. 45-54, 2001.

[8] M. Shirazian, and M. H. Farahi, "Optimal control strategy for a fully determined HIV model,"Intelligent control and automation, pp. 15-19, 2010.

[9] S. P. Banks, "Mathematical theories of nonlinear systems," Prentice Hall, Hertfordshire, 1988. 